\title{
Contrast discrimination by the methods of adjustment and two-alternative forced choice
}

\author{
Donald Laming
}

Published online: 13 September 2013

(C) Psychonomic Society, Inc. 2013

\begin{abstract}
Fifty years after the advent of signal-detection theory, some visual scientists still adhere to the notion of a high, fixed threshold. Indeed, experienced observers are able to deliver consistent settings of thresholds using the method of adjustment, but those settings are different from the thresholds obtained with two-alternative forced choice (2AFC) procedures. This article illustrates the difference by reference to four existing studies of contrast discrimination. All four sets of data show clear subthreshold facilitation, but the method of adjustment produces a rectilinear characteristic that corresponds to the use of different criteria over different ranges of contrast, whereas $2 \mathrm{AFC}$ procedures produce a curvilinear characteristic with generally lower thresholds. This article proposes a theoretical explanation for the difference.
\end{abstract}

Keywords Contrast discrimination - Method of adjustment . Signal detection theory $\cdot$ Subthreshold facilitation $\cdot$ Thresholds

In the nineteenth century, it was supposed that a very weak stimulus would have no effect at all, as it would be below the threshold of sensation . Lotze (1879/1884) expressed the idea as follows:

None of the theories that have been formed on this point [Fechner's law] explain why the continuous curve of growth in the strength of the stimulus is not continuously followed by the slower augmentation in the strength of the sensation, - - why, on the contrary, there remains an interval throughout which the stimulus strengthens without showing any result, until, at last, on its reaching a final degree of strength, it produces an observable difference in the sensation. (Lotze, 1879/1884, p. 455)

D. Laming $(\bowtie)$

Department of Psychology, University of Cambridge, Downing

Street, Cambridge CB2 3EB, England, UK

e-mail: drj1@hermes.cam.ac.uk
Lotze was writing in 1879 , but the idea was current much earlier (see Boring, 1950, pp. 167 and 256).

This is the idea that Swets, Tanner, and Birdsall (1961) endeavored to displace, in particular with a four-alternative second-guess experiment. The signal was a flash of light, 30' of arc in diameter and of 10-ms duration, presented in the middle of a large uniform background of $10 \mathrm{f}$. 1 . Each trial included four observation intervals, with the flash being presented in exactly one of them. The observer was asked to report which interval contained the flash, and then to provide a second guess. If the first response was wrong, then a fortiori the signal failed to exceed threshold, and a second guess (conditional on that first error) must necessarily be blind, with a probability of $1 / 3$ of being correct. Observers guessed correctly systematically above that chance level, and increasingly so with stronger signals (see Swets et al., 1961, Fig. 10).

Notwithstanding that Swets, Tanner, and Birdsall (1961) showed the nineteenth-century notion of a threshold to be false, some visual scientists still adhere to the notion of a high, fixed threshold (Graham, 1989; Laming, 2013). Experienced observers are able to adjust a variable stimulus so that it is just detectable and to set systematically consistent thresholds. The method of adjustment is quick, whereas more rigorous procedures (e.g., 2AFC) would be infeasible over a very large program of observations. Some studies (e.g., Campbell \& Kulikowski, 1966, and Kulikowski, 1969/1973, discussed in the next section) are practicable only by this means.

This article attempts a reconciliation between the practice of determining thresholds by the method of adjustment, and the demonstration by Swets, Tanner, and Birdsall (1961) that even the weakest stimuli have demonstrable effects, by reinterpreting the notion of a threshold. In the first place, the presentation of a stimulus provides the observer with statistical information that may, or may not, be sufficient to justify reporting a detection. The question of what level of information is sufficient is at the observer's disposal (Green \& Swets, 1966). Suppose now that several distinct sensory cues might be independently used, in like manner, to detect the presence of a stimulus. These different cues 
signal different features of the stimulus, and a feature is perceived (and the stimulus detected thereby) if the information provided by the cue exceeds some minimal level (i.e., if the signal exceeds a "threshold"). If the information falls short of this value, the feature in question is not perceived. If none of the stimulus features are perceived, the stimulus is not reported. This, of course, is "probability summation" (Laming, 2013).

If, on the other hand, the observer is required to say whether or not the stimulus was presented and is provided with feedback after each trial, different sources of information may be combined to make that judgment. Such combination leads to a difference in the observed relation between stimulus magnitude and threshold, a difference that is especially apparent in the examples below. The method of adjustment can give systematic results, but those results may differ from those of more rigorous $2 \mathrm{AFC}$ procedures. This article is concerned to explain the difference.

\section{Contrast thresholds by the method of adjustment}

The "method of adjustment" means simply that the observer adjusts the stimulus until it is just detectable and assumes that "just detectable" corresponds to some measurable property of the stimulus. Figure 1 illustrates the configuration of the stimuli (though not to scale) in two experiments by Kulikowski (1969/1973) and Campbell and Kulikowski (1966). A grating of 10 cycles/deg filled a circular field of $2^{\circ}$ diameter, set within a surround of the same space-average luminance. The contrast was switched abruptly between $C$ and $C+\Delta C$ every second (square-wave modulation at 0.5 $\mathrm{Hz}$ ). The $\Delta C$ was adjusted by the observer setting an attenuator, in units of $0.5 \mathrm{~dB}$.

The threshold measurements are displayed in Figs. 2 and 3. The data are presented on logarithmic scales, whereas the inset diagrams reproduce the data for low contrasts on linear scales to exhibit the rectilinear nature of the threshold characteristic. The decrease in threshold for contrasts around the detection threshold (indicated by the vertical arrows) provides an especially incisive characterization of the difference between the methods of adjustment and 2AFC procedures. ${ }^{1}$

Looking at the inset diagrams to Figs. 2 and 3,

1. For $C=0.001$ to 0.006 (0.002 to 0.006 , in Fig. 3 ), the increment in contrast, $\Delta C$, was set so that $C+0.82 \Delta C=$ 0.0083 (or $C+1.34 \Delta C=0.0086$, in Fig. 3). Note that although the observed relation is linear, the adjustments were

\footnotetext{
${ }^{1}$ The data in these two figures have been abstracted from a much larger set of measurements - in Fig. 2 (Kulikowski, 1969/1973) comparing masking of 10 cycles/deg by gratings of different wave numbers, and in Fig. 3 (Campbell \& Kulikowski, 1966) comparing different orientations of a test and a masking grating. Graphical plots of the full sets of data may be seen in Kulikowski (1991, p. 307).
}

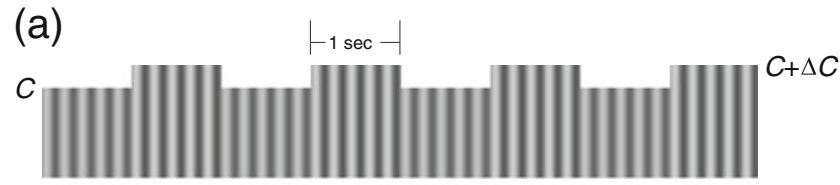

(b)
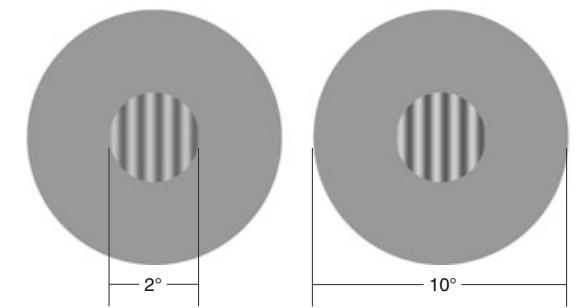

Fig. 1 Configuration of the stimuli (not to scale) in the experiments by Campbell and Kulikowski (1966) and Kulikowski (1969/1973).

geometric $(0.5 \mathrm{~dB})$, so that the linear relation is not an artifact of repeated linear adjustments at arithmetically spaced contrasts. I suggest that the observer set the increment so that the combined contrast could just be seen. ${ }^{2}$ Since the contrast of that combined grating, as adjusted to threshold, was (approximately) independent of $C$, detection cannot have depended on the temporal transition from $C$ to $C+\Delta C$. The grating must have been detected by means of a spatial comparison with the uniform luminance around its boundary.

2. For $C$ greater than 0.008 (0.02 in Fig. 3 ), the grating is always visible, and the observer now has to depend on the temporal change in contrast. The data conform to Weber's law, with ratios of 0.32 and 0.18 , respectively.

3. At contrasts below 0.001 (0.002 in Fig. 3), the grating can be detected at lower levels of $\Delta C$ than would be prescribed by extrapolation of the linear relationships in Criterion 1 above. This improved detection cannot depend on a spatial comparison (the thresholds are too low), and it must therefore depend on the temporal changes at onset and offset. Moreover, at these contrast levels, $\Delta C$ is several times greater than $C$; that is, the detection of $\Delta C$ is limited much more by background noise than by the standard contrast $C$, so that the value of $C$ ceases to affect the threshold. At the transitional contrasts (0.001 and 0.002), both cues - temporal changes at onset and offset and comparison with the matched surround - are available to support discrimination. But the rectilinear character of the relationship implies that only one or the other, and not both in combination, is used at any one time.

The different coefficients ( 0.82 and 1.34) of $\Delta C$ in Figs. 2 and 3 need examination. These two sets of data are for the same observer (Kulikowski) in the same laboratory with the same equipment, the only difference being the mean luminance (75 and $40 \mathrm{~cd} / \mathrm{m}^{2}$, respectively). Nevertheless, the thresholds in Fig. 2 are consistently about $2 \mathrm{~dB}$ higher than their counterparts

\footnotetext{
${ }^{2}$ The question of why $\Delta C$ has different coefficients (0.82 and 1.34) in Figs. 2 and 3 is examined below, and the question of why these coefficients are different from 1.0 later still.
} 


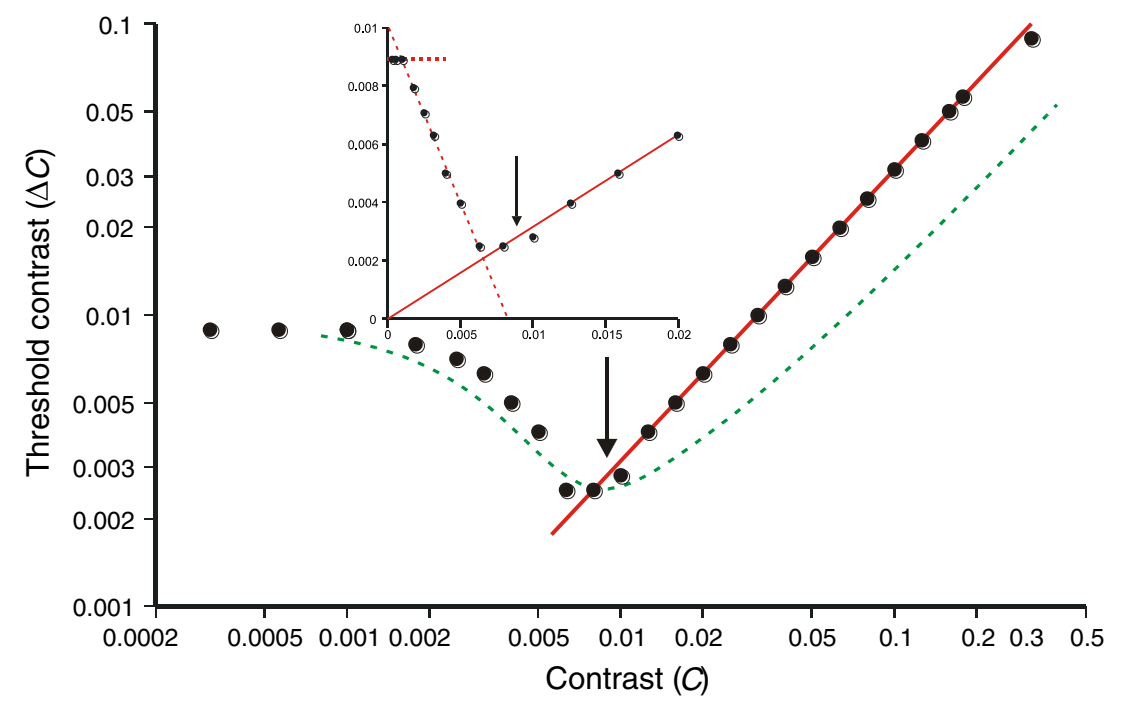

Fig. 2 Difference thresholds for contrast from the experiment by Kulikowski (1969/1973). The data for the lowest contrasts are reproduced on linear coordinates in the inset. The vertical arrows mark the detection threshold on the abscissa for the grating presented continuously. The continuous straight line (red online) is Weber's law, and the broken curve (green online) effects a comparison with what might be expected from

in Fig. 3 (see Table 1), suggesting some error in calibration of the ordinate in one set of data or the other. ${ }^{3}$ The test grating $\Delta C$ and the mask $C$ were produced on two different oscilloscopes and combined optically (Campbell \& Kulikowski, 1966, p. 438). Although this is an error-prone method of creating the particular subset of stimuli of interest here, it is the easiest way when comparing masking by different wave numbers (Kulikowski, 1969/1973), and it is essential when comparing different orientations of the masker (Campbell \& Kulikowski, 1966). But when the test and masking gratings are of the same wave number and orientation, as in the data extracted in Figs. 2 and 3, any lateral misalignment would have introduced a phase shift and attenuated the net increment $\Delta C$. To accommodate the apparent anomaly between these two sets of data, the increments $\Delta C$ in Fig. 2 have been rescaled by $-2 \mathrm{~dB}$ in Table 1 to give the results summarized there.

The rectilinear character of the data in Figs. 2 and 3 points to three distinct sensory cues:

1. the spatial comparison with the uniform surround at contrasts just above the detection threshold,

2. the temporal change in contrast at suprathreshold values, and

3. the temporal onset and offset at detection threshold.

These three cues provide independent sources of information, and the results in Figs. 2 and 3 indicate that the observer uses one or

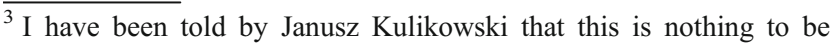
wondered at.
}

2AFC procedures. The data are from Warunki Graniczne Percepcji Wrokowej: Prace Instytutu Automatyki [Limiting Conditions of Visual Perception] (pp. 1-33), by J. J. Kulikowski (Trans. G. B. Henning), 1969/1973, Manchester, UK: P.A.N. The figure is adapted from Sensory Analysis (p. 148), by D. Laming, 1986, London, UK: Academic Press. Copyright 1986 by Academic Press. Reproduced by permission.

another, whichever is the most informative at the contrast in question, but does not combine information from different sources. "Threshold" may now be identified with a criterion level of information (or a signal that exceeds a "threshold") sufficient to support the perception of the stimulus feature to which the cue relates.

\section{Contrast thresholds by $2 \mathrm{AFC}$ procedures}

Nachmias and Sansbury (1974) The subject in this study viewed a 3 cycle/deg grating through a rectangular aperture in a surround matched to the grating in color and mean luminance. On each trial, two separate displays were presented for a 250-ms duration. (Differences in contrast were not juxtaposed, as in Fig. 1 [top], and this was effectively a discrimination between the pairs of gratings in Fig. 1 [bottom], although the stimulus fields were of different shapes.) The two contrasts, $C$ and $C+\Delta C$, were presented in either order, at random. The observer attempted to identify the stimulus of higher contrast $(C+\Delta C)$, whereafter he or she was told which response was correct. $\Delta C$ was varied in a staircase procedure designed to converge on the value that would give $79.6 \%$ correct responses (Weatherill \& Levitt, 1965). The thresholds for three different observers are shown in Fig. 4.

The threshold decreases for contrasts slightly above zero and reaches a minimum (maximum discriminability) at a standard contrast $(C)$ of about 0.004 . This is approximately the detection threshold (downward-pointing arrows) so that maximum discriminability is achieved with one stimulus at the detection threshold and the other slightly greater. The curves in Fig. 4 are calculated from the model below. 


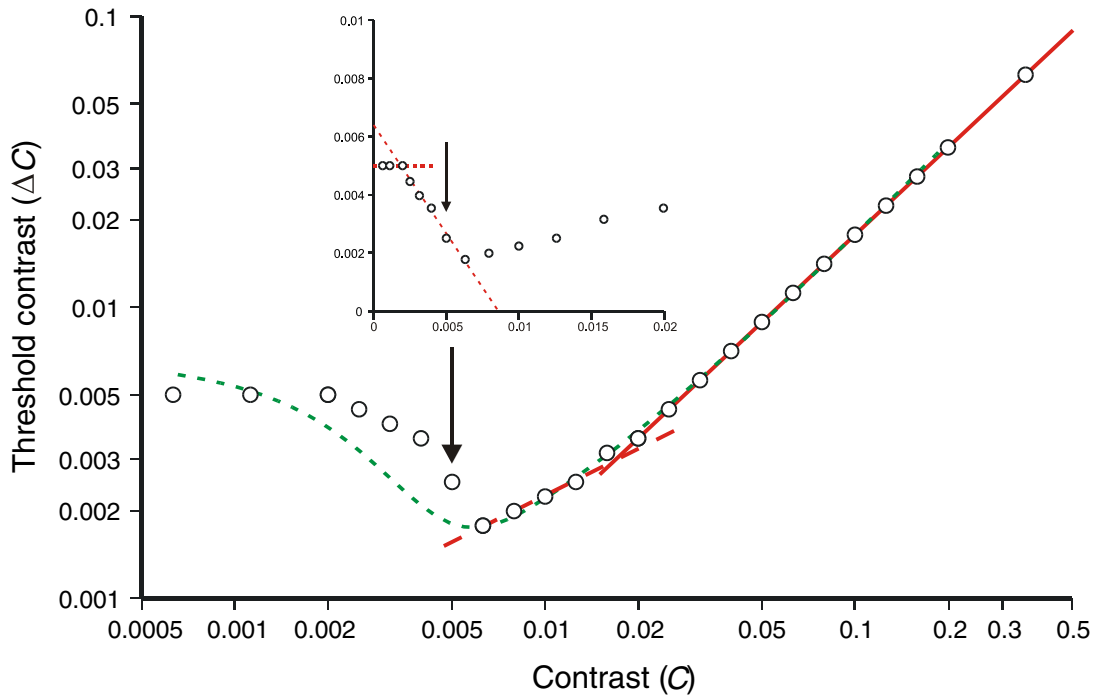

Fig. 3 Difference thresholds for contrast from the experiment by Campbell and Kulikowski (1966). The data for the lowest contrasts are reproduced on linear coordinates in the inset. The vertical arrows mark the detection threshold on the abscissa for the grating presented continuously. The continuous straight line (red online) marks Weber's law, and the broken line (also red online) a square-root relation, whereas the curve (green online) effects a comparison with what might be expected from 2AFC procedures. The data are from "Orientational Selectivity of the Human Visual System," by F. W. Campbell and J. J. Kulikowski, 1966, Journal of Physiology, 187, pp. 437-445. The figure is adapted from Sensory Analysis (p. 146), by D. Laming, 1986, London, UK: Academic Press. Copyright 1986 by Academic Press. Reproduced by permission.
Legge and Foley (1980) The observer viewed a 2-cycle/deg grating through a rectangular aperture, either $6^{\circ}$ wide ("wide field masking") or $0.75^{\circ}$ wide ("narrow field masking"), with a white surround. On each trial, two separate displays were presented for a 200-ms duration. Thereafter, the experimental procedure was the same as in the experiment by Nachmias and Sansbury (1974) above. The thresholds for one observer, but two different field widths, are shown in Fig. 5.

The thresholds for the lowest contrasts in Figs. 4 and 5 are reproduced on linear coordinates in the inset, as in Figs. 2 and 3 above. Although one could, of course, draw straight lines passing reasonably close to the data points, these data do not have the conformity to a rectilinear characteristic seen in the data of Campbell and Kulikowski (1966) and Kulikowski (1969/1973), obtained with the method of adjustment.

\section{Interim summary}

2AFC procedures are robust, in the sense that the observer is only required to try to be correct, and the experimental procedure then determines the limit to the observer's accuracy. The method of adjustment is quite different. The data in Figs. 2 and 3 are means of the observer's subjective judgments, and much depends on the integrity of the observer. This particular observer has delivered highly systematic data, and it is pertinent to enquire how those judgments were made. But another observer might not be so consistent, so the enquiry here concerns the sensory judgments of this particular observer.

Thresholds obtained with the method of adjustment decompose into three (or possibly four; see Fig. 3) linear segments; 2AFC thresholds do not. The method of adjustment could be modeled as three high-threshold units, with different units being used over disjoint ranges of contrast.

For $C \leq 0.006$, the threshold is the minimum of the $\Delta C \mathrm{~s}$ required by Criteria 1 (spatial comparison with the matched surround) and 3 (temporal onset and offset). This is what one should expect from the conjunction of two high-threshold units.

For $C \geq 0.002$, on the other hand, the threshold is the maximum of the $\Delta C$ s required by Criteria 1 (spatial comparison with the matched surround) and 2 (temporal change in contrast at suprathreshold values). It needs to be explained why $\Delta C$ is not adjusted to give $C+0.82 \Delta C=0.0083$ (or $C+1.34 \Delta C=$ 0.0086, in Fig. 3) at all levels of contrast; the physical stimuli,

Table 1 Summary of thresholds in Figs. 2 and 3 by the method of adjustment

Section of Threshold Characteristic

Absolute threshold for temporal change in contrast 0.009

Limit set by comparison with matched surround

Weber fraction
Kulikowski (1969/1973) Kulikowski (1969/1973), with $\Delta C-2$ dB Campbell \& Kulikowski (1966)

$\begin{array}{ll}C .009 & 0.006 \\ C+0.82 \Delta C=0.0083 & C+1.31 \Delta C=0.0083\end{array}$

0.32

0.20
0.005

$C+1.34 \Delta C=0.0086$

0.18 

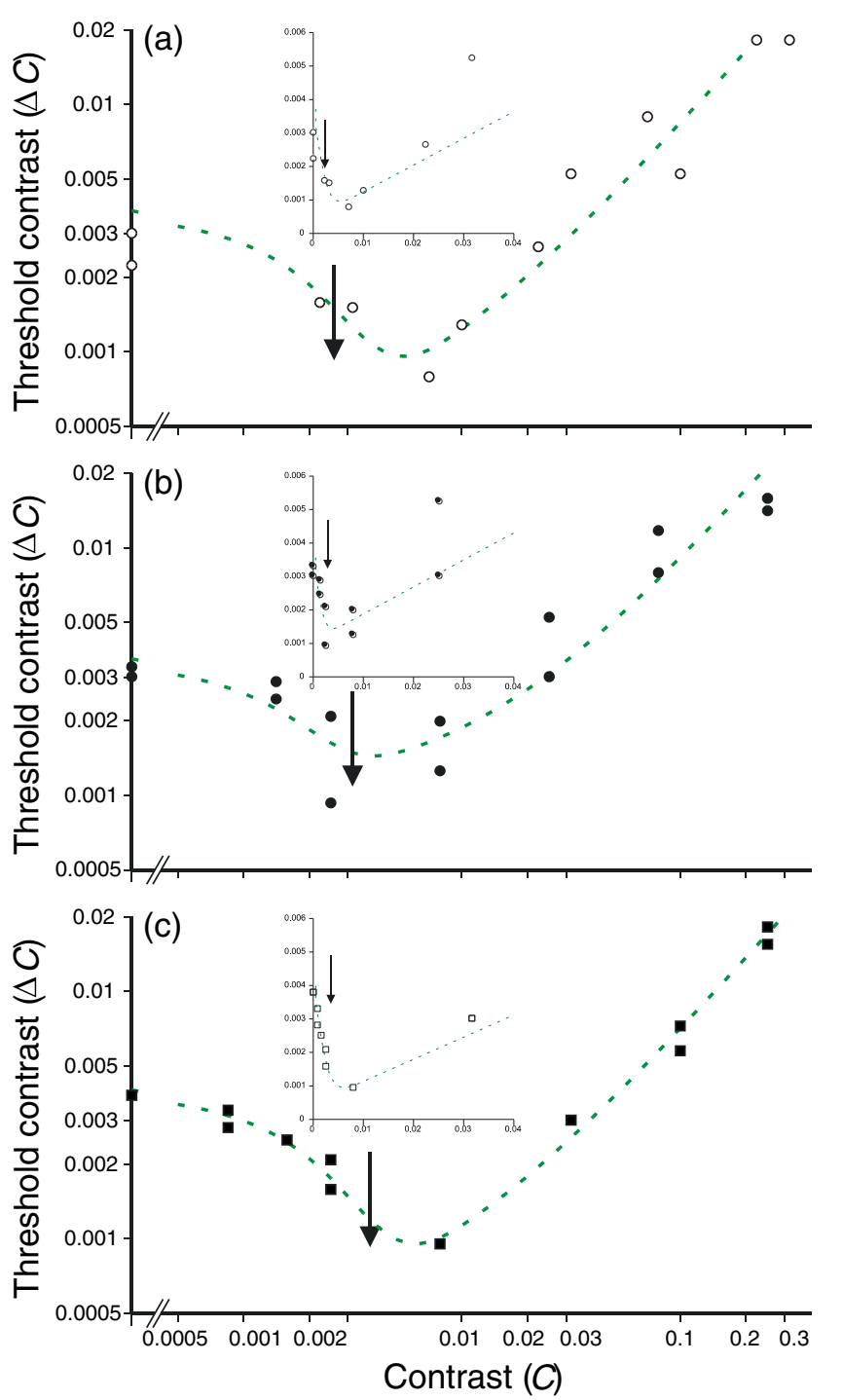

Fig. 4 Difference thresholds for contrasts from three observers in the experiment by Nachmias and Sansbury (1974). The data for the lowest contrasts are reproduced on linear coordinates in the insets. The vertical arrows mark the detection threshold on the abscissa. The curves (green online) represent calculations based on the model in the text. From "Probability Summation-A Critique," by D. Laming, 2013, Journal of the Optical Society of America A, 30, p. 304. Copyright 2013 by the Optical Society of America. Adapted by permission.

with $\Delta C$ in counterphase to $C$, can certainly be produced. Kulikowski (1976, Fig. 6) reported thresholds for both increments and (counterphase) decrements, using the procedure of Fig. 2. Up to $C=0.008$, both increment and decrement thresholds conform approximately to $C \pm \Delta C=$ constant.

For $C \geq 0.008$, the standard contrast $(C)$ is visible all the time, and both threshold units are continuously exceeded. The Weber law section therefore represents the detection of change in contrast, rather than the detection of contrast per se.

The relation $C+1.34 \Delta C=0.0086$ in Fig. 3 (or $C+1.31 \Delta C=$ 0.0083 in Table 2) has been interpreted as approximately setting the combined contrast to a fixed level. So why are the coefficients not equal to 1.0 ? One answer is that $\Delta C$ was switched on and off at $0.5 \mathrm{~Hz}$, and would have been subject to some attenuation in consequence. It is possible that the coefficient 1.34 (or 1.31) simply compensates for this attenuation. If this were so, then setting the combined contrast to a fixed level would be exact.

The $2 \mathrm{AFC}$ procedures in the experiments here included trialby-trial feedback; the method of adjustment, of necessity, does not. It is plausible that feedback directs the observer's attention to all available sources of information [e.g., both Criteria 1 (spatial comparison with the matched surround) and 3 (temporal onset and offset) around $C=0.002$ ], thereby smoothing the rectilinear threshold characteristic. This idea is supported by a comparison between the threshold values in Table 2. Overall, 2AFC procedures appear to give lower thresholds than does the method of adjustment (except for the $0.75^{\circ}$ field in Fig. 5). It may be that "adjustment" equates to a higher degree of certainty than $79.6 \%$. The lower row in Table 2 shows that $2 \mathrm{AFC}$ procedures also afford a smaller minimum threshold, $\Delta C$.

Finally, the method of adjustment gives Weber's law for contrast at suprathreshold contrast levels; $2 \mathrm{AFC}$ procedures do not. This is not obvious from Figs. 4 and 5, but other studies, in particular Kulikowski (1976) and Legge (1981), have shown $\Delta C$ to increase as $C^{0.7}$ (or thereabouts) suprathreshold.

\section{Model for subthreshold facilitation}

The curvilinear subthreshold facilitation characteristics in Figs. 2, 3,4 , and 5 must now be explained. The limited precision of the data means that a variety of models could be proposed. The one that follows here has recently been republished with specific application to the detection of contrast (Laming, 2013) and is merely summarized here, except that the equation of the characteristics is set out in detail.

1. Differential interface Retinal ganglion cells receive both positive (excitatory) and negative (inhibitory) inputs within their receptive fields. The two inputs are statistically balanced, so that the mean inputs cancel. The contrast, however, is merely attenuated, because the positive and negative inputs are differently disposed in space and transmit different depths of modulation. The quantal noise is also transmitted, because the positive and negative inputs are statistically independent. The two noise components combine in square measure to produce an approximately Gaussian noise of power proportional to the luminance, against which the attenuated contrast has to be detected.

2. Half-wave rectification Retinal ganglion cells receive both positive and negative inputs, but transmit action potentials of one polarity only. In terms of radio engineering, this is half-wave rectification. Averaged over the face of the grating, the half-wave-rectified output generates an increment 
Fig. 5 Difference thresholds for contrasts from the experiment by Legge and Foley (1980). The data for the lowest contrasts are reproduced on linear coordinates in the inset. The curves (green online) represent calculations based on the model in the text.

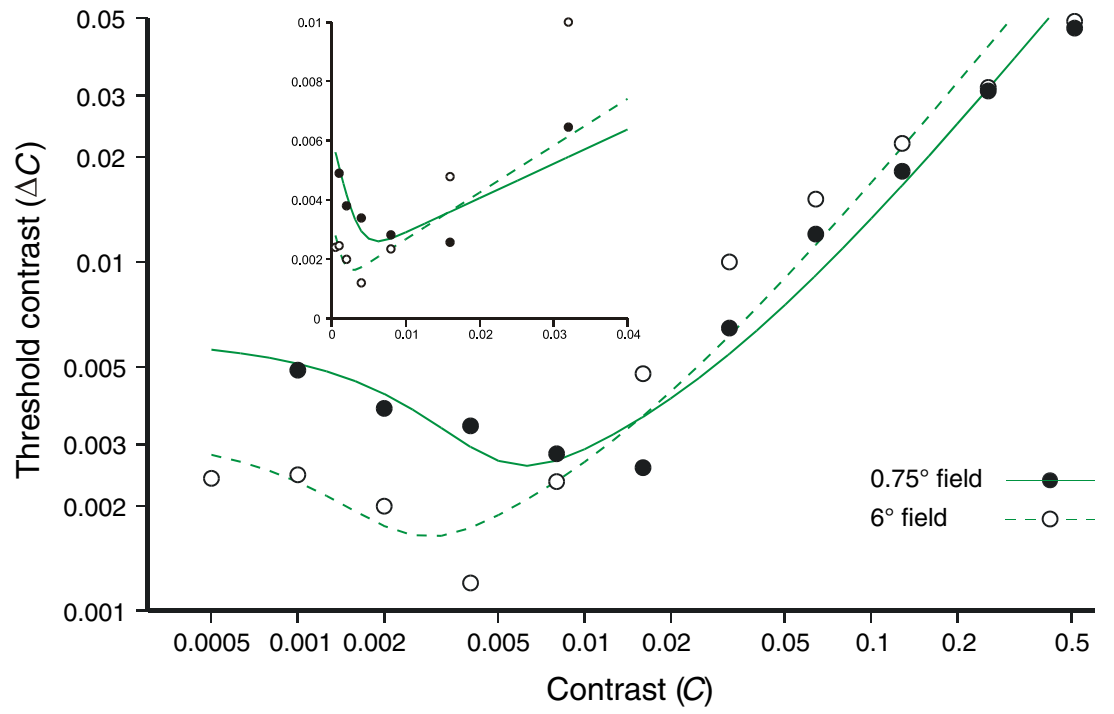

proportional to the square of the contrast for the smallest contrasts, transiting to a linear relation suprathreshold.

3. Second transform in cascade The receptive field organization seen in the retina is repeated at higher levels in the visual pathway. The increment over the face of a grating is detected by comparison with its uniform surround, repeating the analytical process at the retinal level. This cascaded transform is fourth power for the smallest contrasts, transiting to linearity as contrast increases.

4. Discrimination of contrast When a contrast $C+\Delta C$ has to be distinguished from contrast $C$, it is assumed that Weber's law applies to the two quantities output from the second stage above, each superimposed on a noise background; more precisely, that discrimination depends on a comparison of two noise processes with these respective noise powers (Laming, 2010).

Let the net attenuation of contrast be $a$, so that a sinusoidal signal of amplitude $a C$ has to be distinguished from the noise background. Let the output from the retinal ganglion cell, normalized with respect to the noise background, increase as $h[a(C)]$ with respect to contrast. This provides the input to the second stage of analysis (detection of a grating vis-à-vis the matched surround), and the output from this second stage increases as $h\{h[a(C)]\}$. Let $\Delta C_{0}$ be the detection threshold with regard to zero contrast, and let $\Theta$ be the Weber fraction. Then, $h\left\{h\left[a\left(\Delta C_{0}\right)\right]\right\}$ is $\Theta$ times the noise background, and

$h\{h[a(C+\Delta C)]\}=(1+\Theta) h\{h[a(C)]\}+h\left\{h\left[a\left(\Delta C_{0}\right)\right]\right\}$.

This equation describes the curvilinear characteristics in Figs. 2-5. These characteristics realize the assumption that Weber's law applies directly to the cascaded transform.

\section{Weber's law applied to contrast}

As a matter of convenience in calculation, it has been assumed that Weber's law applies to contrast, but this is known not to be true when $2 \mathrm{AFC}$ procedures are used. Discriminations between two separate contrasts have usually shown the difference threshold to increase as the 0.7-0.8 power (see Figs. 4 and 5). This has been replicated, in particular, by Kulikowski (1976; see Laming, 1986, Fig. 1.11, p. 16) and by Legge (1981). Legge compared a variety of different stimulus configurations, two separate 200-ms presentations with and without a continuous background, with and without preadaptation to that background, and with preadaptation to a fixed contrast. The
Table 2 Comparison of threshold values obtained by the two different methods

${ }^{\dagger}$ Estimated from the fit of the model.

${ }^{*}$ Adjusted value from Table 1.

\begin{tabular}{|c|c|c|c|c|}
\hline Study & $\begin{array}{l}\text { Nachmias and } \\
\text { Sansbury (1974) }\end{array}$ & $\begin{array}{l}\text { Legge and } \\
\text { Foley (1980) }\end{array}$ & $\begin{array}{l}\text { Campbell and } \\
\text { Kulikowski (1966) }\end{array}$ & $\begin{array}{l}\text { Kulikowski } \\
(1969 / 1973)\end{array}$ \\
\hline \multirow[t]{3}{*}{ Detection threshold } & 0.0026 & $0.0033^{\dagger}$ & \multirow[t]{3}{*}{0.005} & 0.0089 \\
\hline & 0.0033 & $0.0061^{\dagger}$ & & $(0.006)^{\ddagger}$ \\
\hline & 0.0038 & & & \\
\hline \multirow{3}{*}{$\begin{array}{l}\text { Minimum difference } \\
\text { threshold }\end{array}$} & 0.0008 & 0.0012 & \multirow[t]{3}{*}{0.0018} & \multirow{3}{*}{$\begin{array}{l}0.0025 \\
(0.0020)^{\ddagger}\end{array}$} \\
\hline & 0.0014 & 0.0025 & & \\
\hline & 0.0010 & & & \\
\hline
\end{tabular}


exponents for wave numbers between 2 and 8 cycles/deg are "relatively impervious" to all of these configurational manipulations.

If it be accepted that parallel channels are tuned to specific wave numbers (Blakemore \& Campbell, 1969; Campbell \& Robson, 1968; Laming, 1991), then a grating will be detected most sensitively by whichever channel is tuned to its wave number. But as the contrast increases, so a grating will engage an increasingly wider range of channels, tuned to adjacent wave numbers, providing an increasing number of parallel "observations." Combining these parallel observations statistically, it is to be expected that the difference threshold will increase less rapidly than Weber's law prescribes (Laming, 2013). Thus, the apparent conformity to Weber's law in Figs. 2 and 3 is consequent on the observer focusing on the temporal transition between contrasts $C$ and $C+\Delta C$. Conformity to Weber's law means that the discrimination threshold increases more rapidly than in Figs. 4 and 5 (exponents 0.75 and 0.8, respectively); that is to say, the cues used in Figs. 2 and 3 are less informative than the combination of cues in Figs. 4 and 5. The abrupt switching of contrast confers no advantage at all.

\section{Discussion}

The stimulus configuration at the bottom of Fig. 1 affords three cues to discrimination:

1. spatial comparison with the uniform surround at contrasts just above detection threshold,

3. temporal onset and offset at the detection threshold, and

4. direct comparison between the two contrasts.

The top of Fig. 1 adds:

2. temporal alternation at $0.5 \mathrm{~Hz}$ between the contrasts $C$ and $C+\Delta C$.

The rectilinear characteristics in Figs. 2 and 3 indicate that the observer has used first Cue 3, then Cue 1, and finally Cue 2, within disjoint ranges of contrast. Direct comparison between the two contrasts (Cue 4) does not figure at all. The curvilinear characteristics in Figs. 4 and 5, on the other hand, are calculated from a sensory-analytic model that begins with a physical model of the stimulus and, for that reason, arguably combines all sources of information in one decision variable. This interpretation is supported by the $0.7-0.8$ power law for suprathreshold contrasts; Cue 4 turns out to be more informative than Cue 2, notwithstanding that it is not used by the observer of Figs. 2 and 3 .

An experienced observer can deliver systematic data with the method of adjustment. Only one cue is used at a time, and the data in Figs. 2 and 3 correspond, in effect, to detection thresholds for the different stimulus features. "We consider as the threshold contrast such a contrast at which the subject notices a change in the course of the periodical appearance and disappearance of the pattern on the screen for half the number of presentations" (Kulikowski, 1973, p. 13). Thus, the classical notion of a threshold is meaningful in the sense of consistent adjustments made by an experienced observer. But with a different experimental paradigm, the same observer could attend to subthreshold stimuli and produce data like those in Figs. 4 and 5.

What about the alternative? King-Smith and Kulikowski (1981), applying the probability summation model to the detection of bright lines and discrimination between the presentation of one line or two, concluded, from their attempts to model the psychometric functions, that "the experimental data are not consistent with this idea [high threshold], but they are consistent with 'low-threshold' processes-i.e., barriers which are low enough to allow transmission of occasional peaks of visual noise" (p. 243). That is, some false positive reports cannot be attributed to guessing when the threshold is not exceeded.

This is functionally a different idea from a "high threshold." The "high threshold" is an alternative to signal-detection theory and has long been known to be wrong. A "low threshold" is an alternative to a nonlinear transform of low contrasts (e.g., the two square-law transforms in cascade above). It is unclear whether a "low threshold" can account, for example, for subthreshold facilitation. This suggestion has been made (King-Smith, 2005), but no model has yet been worked out to the point that comparison can be made with experimental data.

It is also unclear whether King-Smith and Kulikowski's (1981) "low-threshold" processes would have any impact on the adjustments in Figs. 2 and 3. A "low threshold," in the sense of Swets, Tanner, and Birdsall (1961, p. 324) or Solomon (2007, Fig. 2), would leave much of the noise distribution suprathreshold. The adjusted contrast in Figs. 2 and 3 would likewise have to be suprathreshold to afford $50 \%$ detection. However, all of these authors have overlooked this problem.

Appeal to any kind of fixed threshold or barrier, whether high or low, poses this question: What does the barrier apply to-luminance, incremental luminance, contrast, or what? If it is luminance, then the idea can only apply at absolute threshold (Koenig \& Hofer, 2011). If it is incremental luminance, there has to be some way of stripping the increment from the background prior to the barrier (e.g., the differential interface above); but a low threshold is not then needed, because a suitable nonlinear transform results from the interface operation. If it is contrast, then the input has also to be scaled in inverse proportion to the ambient luminance (so that the threshold applies specifically to contrast, not to Contrast $\times$ Luminance). In practice, the notion of a fixed threshold, whether high or low, is applied to whatever stimulus measure the theorist is playing with, without regard for this problem. But without an answer to this question, a "low- 
threshold" model, or indeed any model that does not specify how contrast is extracted from the luminance signal, suffers from a serious deficit in explanation.

This brings the discussion back to the problem that this article is addressing: how to reconcile data from the method of adjustment (Figs. 2 and 3) with those from 2AFC methods (Figs. 4 and 5). The $2 \mathrm{AFC}$ data reveal more information than is apparent in thresholds by adjustment, but, if that additional information be ignored, an experienced observer can systematically adjust a stimulus so that some selected feature is just noticeable.

The phenomenon of subthreshold facilitation results from a nonlinear input transform, such as Eq. 1 above. Experimental data on the discrimination of contrast indicate that the nonlinear input transform is approximately fourth power for the very smallest contrasts, and transits to a linear law for suprathreshold contrasts (Laming, 2013). Intermediate in that transition, it will be approximately square-law, and this is probably the source of the approximately square-root relation for contrasts from 0.006 to 0.02 in Fig. 3. Note that Eq. 1 (the (green online) dashed curve in Fig. 3) gives an accurate fit to the thresholds within this range of contrasts.

This is quite distinct from the much simpler idea of "subthreshold summation" (Kulikowski, 1991, pp. 304-305). If stimuli below a detection threshold contribute nothing to discrimination, and only the stimulus that can be seen is material, then, in the presence of a subthreshold stimulus, it is sufficient to increase that stimulus up to the threshold value. It is exactly Lotze's (1879/1884) idea. This makes subthreshold summation an interaction within the physical stimulus, not within sensory analysis. But it also implies the resolution of arbitrarily fine differences with respect to a stimulus level just below detection threshold, and we know that input noise precludes such a resolution. "Subthreshold summation" has been interpreted here as an adjustment of the stimulus so that some particular feature is just detectable, whereas other (subthreshold) features are disregarded.

For two reasons, the method of adjustment should lead to higher threshold measurements. First, the objective methods included the provision of immediate feedback - which is not essential to 2AFC procedures, but was incorporated in the two studies examined here. Feedback, as has already been suggested, drives the observer to the most effective use of sensory information that is feasible. The method of adjustment applies no such pressure.

Second, the information utilized in Figs. 2 and 3 is information about particular features of the stimulus. The stimulus is seen as it is to the extent that sufficient evidence is available for each individual feature. That is, the method of adjustment focuses on an individual feature of the stimulus, and neglects information about other features of the same stimulus, notwithstanding that that neglected information is discriminative. So, an objective staircase procedure might deliver a lower threshold by driving the observer to make use of all available information. In this way, observers can make discriminative responses of demonstrably greater-than-chance accuracy about stimuli that they say they cannot see.

We do not know what proportion of identifications would be correct, comparing a standard contrast with a level that was "just noticeably different" by the method of adjustment, but the comparison of detection thresholds in Table 2 provides some indication. Complications in this comparison arise from differences in the levels of retinal illuminance and in the wave numbers of the gratings used. Both of these factors affect detection thresholds. Without going into details, it is possible to calculate the retinal illuminance and make comparison with the comprehensive set of threshold measurements for gratings published by van Nes (1968, p. 34). Using van Nes's measurements for green light $(\lambda=525 \mathrm{~nm})$, the threshold values all come out about the same: 0.0025 to 0.0027 for a "just not noticeable" value of contrast, and 0.0032 to 0.0036 for a "just noticeable" value. On this basis, the differences in retinal illuminance and also in wave number can be ignored, and Table 2 sets out the unadjusted values of the detection thresholds. The objective staircase methods give lower thresholds, especially since, in the studies by Nachmias and Sansbury (1974) and by Legge and Foley (1980), the stimuli were respectively presented for 0.25 and $0.2 \mathrm{~s}$ only, as compared with $1 \mathrm{~s}$ (alternation at $0.5 \mathrm{~Hz}$ ) in the other two studies. It therefore appears that the method of adjustment delivers higher threshold measurements than do objective methods, although it should be noted that the apparent differences might be attributable to different observers. Only a rigorous comparison could resolve this issue.

Author note I thank Janusz Kulikowski and the late Fergus Campbell for the use of their data in Figs. 2 and 3. This article is based on a presentation at the EMPG meeting in Pamplona in August 2012.

\section{References}

Blakemore, C., \& Campbell, F. W. (1969). On the existence of neurones in the human visual system selectively sensitive to the orientation and size of retinal images. Journal of Physiology, 203, 237-260.

Boring, E. G. (1950). A history of experimental psychology (2nd ed.). New York, NY: Appleton-Century-Crofts.

Campbell, F. W., \& Kulikowski, J. J. (1966). Orientational selectivity of the human visual system. Journal of Physiology, 187, 437-445.

Campbell, F. W., \& Robson, J. G. (1968). Application of Fourier analysis to the visibility of gratings. Journal of Physiology, 197, 551-566.

Graham, N. S. (1989). Visual pattern analyzers. New York, NY: Oxford University Press.

Green, D. M., \& Swets, J. A. (1966). Signal detection theory and psychophysics. New York, NY: Wiley.

King-Smith, P. E. (2005). Threshold nonlinearities and signal detection theory. Perception, 34, 941-946. doi:10.1068/p5223

King-Smith, P. E., \& Kulikowski, J. J. (1981). The detection and recognition of two lines. Vision Research, 21, 235-250. doi:10.1016/ 0042-6989(81)90117-6

Koenig, D., \& Hofer, H. (2011). The absolute threshold of cone vision. Journal of Vision, 11(1), 21. doi:10.1167/11.1.21 
Kulikowski, J. J. (1973). Warunki graniczne percepcji wzrokowej: Prace Instytutu Automatyki [Limiting conditions of visual perception] (Trans. G. B. Henning, pp. 1-33). Manchester, UK: P.A.N. (Original work published 1969)

Kulikowski, J. J. (1976). Effective contrast constancy and linearity of contrast sensation. Vision Research, 16, 1419-1431. doi:10.1016/ 0042-6989(76)90161-9

Kulikowski, J. J. (1991). What really limits vision? Conceptual limitations to the assessment of visual function and the role of interacting channels. In J. J. Kulikowski, V. Walsh, \& I. J. Murray (Eds.), Vision and visual dysfunction (Limits of visual perception, Vol. 5, pp. 286329). London, UK: Macmillan.

Laming, D. (1986). Sensory analysis. London, UK: Academic Press.

Laming, D. (1991). Spatial frequency channels. In J. J. Kulikowski, V. Walsh, \& I. J. Murray (Eds.), Vision and visual dysfunction (Limits of visual perception, Vol. 5, pp. 97-105). London, UK: Macmillan.

Laming, D. (2010). Fechner's Law: Where does the log transform come from? Seeing and Perceiving, 23, 155-171. doi:10.1163/187847510X503579

Laming, D. (2013). Probability summation - A critique. Journal of the Optical Society of America A, 30, 300-315. doi:10.1364/JOSAA.30.000300

Legge, G. E. (1981). A power law for contrast discrimination. Vision Research, 21, 457-467. doi:10.1016/0042-6989(81)90092-4
Legge, G. E., \& Foley, J. M. (1980). Contrast masking in human vision. Journal of the Optical Society of America, 70, 1458-1471. doi:10. 1364/JOSA.70.001458

Lotze, H. (1884). Metaphysik; Drei Bücher der Ontologie, Kosmologie und Psychologie (Trans. B. Bosanquet). Oxford, UK: Oxford University Press, Clarendon Press. (Original work published 1879)

Nachmias, J., \& Sansbury, R. V. (1974). Grating contrast: Discrimination may be better than detection. Vision Research, 14, 1039-1042. doi: 10.1016/0042-6989(74)90175-8

Solomon, J. A. (2007). Intrinsic uncertainty explains second responses. Spatial Vision, 20, 45-60. doi:10.1163/156856807779369715

Swets, J. A., Tanner, W. P., \& Birdsall, T. G. (1961). Decision processes in perception. Psychological Review, 68, 301-340. doi:10.1037/ h0040547

van Nes, F. L. (1968). Experimental studies in spatiotemporal contrast transfer by the human eye. University of Utrecht: Unpublished doctoral thesis.

Weatherill, G. B., \& Levitt, H. (1965). Sequential estimation of points on a psychometric function. British Journal of Mathematical and statistical Psychology, 18, 1-9. doi:10.1111/j.2044-8317.1965. tb00689.x 